\title{
Potential Role of Endometrial Scratching in Improving Pregnancy Rates Following Intra Uterine Insemination- A Short Commentary
}

\author{
Kulvinder Kochar Kaur*1, Gautam Allahbadia ${ }^{2}$ and Mandeep Singh ${ }^{3}$ \\ ${ }^{1}$ Scientific Director, Centre for Human Reproduction, India \\ ${ }^{2}$ Scientific Director, Rotunda-A Centre for Human reproduction, India \\ ${ }^{3}$ Consultant Neurologist, Swami Satyanand Hospital, India
}

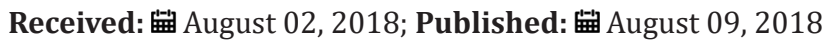

*Corresponding author: Kulvinder Kochar Kaur, Scientific Director, Centre for Human Reproduction, Jalandhar, Punjab, India

\section{Short Communication}

Implantation is the rate limiting step for success during In Vitro Fertilization (IVF). Lot of advances have been done for improving the success rate in IVF, like use of Preimplantation Geneting Screening (PGS) for ensuring euploid embryo transfers besides freeze all cycles for improving embryo-endometrial synchrony, still for fresh cycles implantation remains a question mark.

Endometrial Scratching (ES) or endometrial injury, trauma or biopsy is a fast phenomenon i.e minimally invasive outdoor procedure that causes minimal discomfort. Hysteroscopy can be used to perform it or one can use a lot of soft and rigid endometrial biopsy devices. Since it is commonly performed in the luteal phase, disruption of any early pregnancy is the only big risk associated with this method. Mechanism of action for increasing endometrial receptivity with ES are i) it facilitates endometrial decidualization ii) Because of injury there is healing which=>activation of an immune response, with cytokines, interleukins, growth factors, macrophages and natural killer cells iii) It causes slowing of endometrial maturation to improve synchrony [1]. Though these hypothesis remain untested till date some reasoning is given regarding how ES benefits.

In 2015there was a Cochrane analysis done on 14 Randomized Control Trials (RCT's) which had 2128 IVF patients and it was concluded that ES done in the preceding luteal phase or early follicular phase of a fresh cycle improved the clinical pregnancy rates significantly (relative risk 1.34, 95\% CI-1.21-1.61; P=0.02) in 13 studies [1]. In the only study where ES was done on the day of retrieval was stopped early as it showed a marked reduction in pregnancy rates. However, the results might have been secondary to greater degree of endometrial injury caused by a novak curette. In toto many studies had a high risk of bias along with no uniformity in terms of patient selection, stimulation protocol along with timing of ES, and thus effectivity of ES was just based on moderate evidence.
It is proposed that same ES might effect the embryo implantation in a natural cycle along with IUI cycles. In a survey of 143 doctors in Australia, New Zealand and UK fertility clinics $83 \%$ of those responding to the survey recommended ES for IVF, but only $4 \%$ recommended the same for patients trying to have a pregnancy by natural method or with the use of an IUI [2]. In 2016 another Cochrane review was done but this time regarding nine RCT's which included 1512 patients for timed intercourse and IUI and ultimately concluded that not much evidence of benefit for ES in this setting was there [3]. Following that Cochrane review there have been 4 additional RCT's. Recently Vitaglino et al presented an updated systematic review and meta-analysis where they included 4 additional studies and they only selected 8 studies for final analysis. Contrary to the 2016 Cochrane review Vitaglino et al only used patients that received Ovulation induction along with IUI so that heterogeneity was minimized. Further they excluded the nonpeer reviewed studies which were present in the Cochrane review. Still they had 1871 cycles to be analyzed. They found that Clinical Pregnancy Rate (CPR) along with Ongoing Pregnancy Rate (OPR) were significantly higher having an odds ratio (OR 2.27; $\mathrm{P}<0.00001$ ) and OR 2.04; $\mathrm{P}=0.004$ ), with no increase in multiple pregnancy, abortions or ectopic pregnancy rates. Importantly timing of the ES affected the success of the procedure. If ES was done in the cycle prior to the IUI cycle the CPR, OPR were no different from the control group. In follicular phase procedure IUI was associated with a higher CPR (OR 2.57; $\mathrm{P}<0.00001)$ and OPR (OR 2.27; P=0.0004) [4].

Although they concluded that overall evidence is low in view of high risk of bias in many studies along with heterogeneity in studies. Also patient selection varied regarding cause of infertility; namely unexplained infertility, mild male factor, endometriosis and ovulation dysfunction and variable techniques of ovulation 
induction; clomiphene citrate or letrozole with or without FSH/ HMG, variation in ES technique with use of pipelle, endocell, neonatal feeding tube ,cervical mucus aspiration catheter, Karmen cannula, cyto brush or novak curette, along with timing of ES i.e., follicular or luteal phase of the preceding cycle or early to midfollicular phase or 24-36h before IUI. Thus findings showing that ES in same cycle as IUI during early follicular phase improved both the CPR and OPR, should be the motive to further study and find the exact timing of ES, along with keeping other variables constant like indication of IUI, Technique of ovulation induction, instrument used for ES to be able to make a proper decision on the role of ES in improving implantation along with OPR with the use of ES. Although currently we use it rarely only in IVF failures, we intend to study this very easy, cheap, non-time-consuming method to see if ES might offer any help in cases of IUI with specific indications.

\section{ISSN: 2574-1241}

DOI: 10.26717/BJSTR.2018.07.001547

Kulvinder Kochar Kaur. Biomed J Sci \& Tech Res

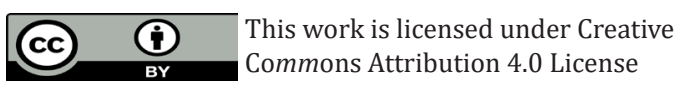

Submission Link: https://biomedres.us/submit-manuscript.php

\section{References}

1. Nastri CO, Lensen SF, Gibreel A, Rene-Fenning RA, Bhattachrya S, et al. (2015) Endometrial injury in women undergoing assisted reproductive technology. Cochrane Database Syst Rev 7: CD009517.

2. Lensen SF, Sadler A, Farquhar C (2016) Endometrial Scratch in for subfertility: Everyone's doing it. Hum Reprod 31(6): 1241-1244.

3. Lensen SF, Manders M, Nastri CO, Gibreel A, Martins WP, et al. (2016) Endometrial injury for pregnancy following sexual intercourse or intrauterine insemination. Cochrane Database Syst Rev 6: CD0011424.

4. Vitaglino A, Noventa M, Seccone G, Gizzo S, Vitale SG, et al. (2017) Endometrial Scratch injury before intrauterine insemination: Is it time to reevaluate its value? Evidence from systematic review and metaanalysis of randomized controlled trials. Fertil Steril 109(1): 84-96.

Assets of Publishing with us
$\begin{array}{ll}\text { BIOMEDICAL } \\ \text { RESARCHES }\end{array}$
- Immediate, unrestricted online access
https://biomedres.us/

